\title{
Pre and postoperative deficiencies in iron, ferritin and B12 vitamin among portuguese patients undergoing bariatric surgery
}

\author{
Mariana Santos Lopes ${ }^{1}$, Bruno M. P. M. Oliveira ${ }^{2,3}$, Olga Neves ${ }^{2,4}$, Diva Melim ${ }^{5}$, \\ Paula Freitas ${ }^{6,7}$, AMTCO Group ${ }^{5}$ and Flora Correia 1,5 \\ ${ }^{1}$ Faculdade de Ciências da Nutrição e Alimentação da Universidade do Porto, Porto, Portugal, \\ ${ }^{2}$ Faculdade de Ciências da Nutrição e Alimentação da Universidade do Porto, Porto, Portugal, \\ ${ }^{3} L I A A D$, INESC TEC, Porto, Portugal, \\ ${ }^{4}$ Sociedade Portuguesa de Ciências da Nutrição e Alimentação, Porto, Portugal, \\ ${ }^{5}$ Centro Hospitalar São João, E.P.E, Porto, Portugal, \\ ${ }^{6}$ Departamento de Endocrinologia, Diabetes e Metabolismo, Centro Hospitalar São João, E.P.E, Porto, Portugal and \\ ${ }^{7}$ Faculdade de Medicina da Universidade do Porto, Porto, Portugal
}

\begin{abstract}
Introduction: According to the World Health Organization obesity it's the result of an abnormal or excessive body fat accumulation, which presents a high risk for the health. Bariatric surgery appears as an alternative to the conventional treatment for the morbid obese individuals. However, this type of intervention causes changes in the anatomy and physiology of the gastrointestinal tract, which may lead to the development of nutritional deficiencies in patients, in particular anemia.
\end{abstract}

Aim: To evaluate micronutrient deficiencies in patients submitted to bariatric surgery in preoperative and postoperative periods, in particular iron, ferritin, and B12 vitamin.

Methods: In this longitudinal study, we evaluated, retrospectively and prospectively, patients who attended the nutrition appointment at a central hospital. We completed a preexisting database containing anthropometric and biochemical data, adding biochemical data, at various periods: pre at 6 th, 12th, 18th, 24th, 30th and 36th months post-surgery.

Results: from the 121 patients submitted to bariatric surgery, 79,3\% were female. The prevalence or iron deficiency reached $15 \%$, ferritin deficiency reached almost $10 \%$ and B12 vitamin deficiency reached $18 \%$. Furthermore there were deficiencies in other nutrients, e.g., vitamin D, magnesium and zinc. There was more than $85 \%$ adhesion to take the multivitamin supplementation and frequent use of specific supplementation.

Conclusion: The prevalence of nutritional deficiencies is high, with a tendency to persist over time even with use of multivitamin supplementation, leading to the need for complementary supplementation, in particular to prevent the risk of anemia. Hence, periodic and long term monitoring is fundamental.

\section{Conflict of Interest}

There is no conflict of interest. 